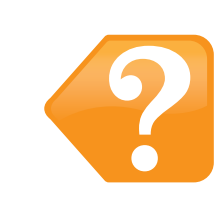

\title{
Generalidades del diseño instruccional
}

\author{
Jorge Enrique Tarazona Suárez ${ }^{1}$
}

Recibido: marzo 15 de 2012. Aprobado: mayo 23 de 2012

\section{Resumen}

Este artículo muestra de forma general lo que es un diseño instruccional, sus soportes teóricos, etapas, elementos y recomendaciones para la implementación en cursos en línea. Se presenta como una propuesta teórica teniendo en cuenta que la instrucción permite una reflexión de orden conceptual con relación al diseño instruccional, como un término utilizado en la actualidad y como un proceso para la elaboración de materiales educativos en ambientes virtuales de aprendizaje.

Se busca que el lector reflexione de manera crítica sobre el significado del diseño instruccional y a su vez encuentre sus beneficios y limitaciones dentro del ámbito educativo. Además se muestra cómo el diseño instruccional es un proceso que integra los contenidos de un curso, los métodos de instrucción, las actividades y los recursos tecnológicos facilitando el aprendizaje de los estudiantes.

Palabras clave: diseño, instrucción, aprendizaje, recursos tecnológicos, contenidos, modelos, objetivos, metodología, evaluación.

\section{Abstract}

This article shows, in a conceptual way, the Instructional Design, its theoretical base, phases, elements and recommendations for the implementation of online courses.

It is presented as a theoretical proposal considering that the instruction allows a conceptual reflection regarding instructional design, as a term used today and as a process for the preparation of educational materials in virtual learning environments.

The goal is for the reader to critically reflect on the meaning of Instructional Design and in turn find its benefits and limitations in the field of education. Also, it is shown how the Instructional Design is a process that integrates course contents, instructional methods, activities and technological resources to facilitate learning of students.

Keywords: design, instruction, learning, technology resources, content, models, objectives, methodology, assessment.

1 Magister en Tecnología educativa (TEC Monterrey). 


\section{INTRODUCCIÓN}

Las nuevas formas de enseñanza y aprendizaje avanzan vertiginosamente con el uso de las tecnologías de la información y las comunicaciones, llevando a replantear el quehacer del docente y sus relaciones con estos medios, conduciéndolo a desarrollar otras habilidades y actualizaciones sobre esas nuevas tecnologías.

A través del diseño instruccional se plantea que los docentes incorporen en el proceso del diseño didáctico de sus asignaturas, el uso de las nuevas tecnologías de la información y la comunicación mediante secuencias didácticas, permitiendo el desarrollo de actividades que fortalecerán el proceso enseñanza -aprendizaje.

Se debe tener en cuenta que el diseño instruccional es un proceso pedagógico en donde se planifica y se produce gran cantidad de material educativo que se adapta a los objetivos propuestos en cada curso, llevando al estudiante a un aprendizaje agradable y de calidad.

El diseño instruccional genera los pasos a seguir de forma sistemática, basándose en las diferentes teorías del aprendizaje, incorporando así elementos fundamentales a través de indicaciones en donde se incluye el análisis de los participantes, la búsqueda de objetivos, las metas y la implementación de estrategias de evaluación.

\section{DEFINICIÓN DE DISEÑO INSTRUCCIONAL}

El diseño instruccional se puede definir como un proceso pedagógico para armar y componer de forma estratégica, planificada y estructurada, los diferentes elementos de un curso en línea, tales como temas, contenidos, actividades, recursos de apoyo y evaluaciones. Esto permite hacer más amigable el aprendizaje en los estudiantes, y hacer el seguimiento necesario para alcanzar los objetivos propuestos.

También se puede definir el diseño instruccional a partir del concepto de diseño de aprendizaje (Sicilia, 2007), no como una solución de los diferentes problemas educativos, sino como un instrumento que requiere de una evaluación y retroalimentación constante, cuyos resultados de evaluación demandará adaptaciones a nuevas circunstancias de ambientes de aprendizaje, teniendo en cuenta las características y las necesidades de los estudiantes, contextualizando así el proceso educativo.

El diseño instruccional es un proceso dinámico e interactivo basado en teorías y enfoques del proceso enseñanza - aprendizaje, que permitirá al docente y al estudiante alcanzar las metas y objetivos planteados.
Sin embargo, antes de realizar el diseño instruccional se debe hacer un listado de las necesidades y las metas educativas a cumplir, para luego diseñar las estrategias que permitan alcanzar los objetivos planteados. Esto, sin dejar de lado la tecnología educativa, entendida como el uso educativo de los medios audiovisuales (Unesco, 1984) o como una relación entre la psicología del aprendizaje, el enfoque sistémico y los medios de comunicación (Chadwick, 1978).

Aguilar (2004) define el diseño instruccional como "un proceso que apoya un enfoque sistemático, organiza de forma sistemática un conjunto de componentes de naturaleza instruccional, que permite satisfacer necesidades y metas instruccionales”. (p.2).

Por su parte, Polo (2001) lo concibe como un momento del proceso arquitectónico de la planificación de la enseñanza...El diseño instruccional involucra prever, organizar y ofrecer pautas para el logro de aprendizajes por parte del estudiante" (p. 10).

Lo anterior lo complementa Poleo (2003), al indicar que el "diseño instruccional es el desarrollo sistemático de especificaciones instruccionales empleando teorías de indicaciones y del aprendizaje para lograr un proceso de enseñanza y aprendizaje de calidad. Abarca el proceso de análisis de la audiencia y de los propósitos de aprendizaje y el desarrollo de un sistema para satisfacer las necesidades". (p.61).

En suma a las definiciones, existen diferentes modelos de diseño instruccional como el denominado ARCS4, conocido también como modelo de diseño motivacional para el aprendizaje y que sirve para actuar en unión con otros modelos de diseño de la instrucción tradicionales (Keller, 1987).

\section{III.SOPORTE TEÓRICO DEL DISEÑO INSTRUCCIONAL}

El diseño instruccional está soportado en cuatro pilares, los cuales le dan fundamento y coherencia: teoría general de sistemas, teoría de la comunicación, teorías del aprendizaje y modelos conceptuales de instrucción.

\subsection{Teoría general de sistemas}

La teoría general de sistemas es un todo organizado y complejo (Kendall, 1991), que describe características tales como orden, propósitos, procesos, funciones, interacción entre elementos, contexto, coherencia, dinámica, objetivos claros y definidos.

A través del enfoque de la teoría general de sistemas se pueden asegurar los resultados y logros que se plantean en un curso determinado (Bertalanffy, y Wein- 
berg, 1987), desarrollando las actividades que forman parte de las etapas del diseño instruccional, basándose en las teorías de enseñanza aprendizaje, con el uso de las tecnologías de la información y de la comunicación aplicadas de forma metodológica y sistemática.

\subsection{Teoría de la comunicación}

La teoría de la comunicación muestra los procesos de transmisión y de información de forma estructurada y sus efectos en el intercambio de mensajes (Cervera, 2005).

La teoría de la comunicación es utilizada dentro del diseño instruccional como medio para desarrollar actividades de instrucción (Fernández y Martínez, 1999).

\subsection{Teorías del aprendizaje}

Las teorías del aprendizaje son de gran influencia en el diseño instruccional ya que tienen estrecha relación con el aprendizaje humano, el fin del diseño instruccional es utilizar estas teorías para generar situaciones en las cuales el estudiante pueda aprender de forma agradable y descomplicada. El diseño instruccional utiliza los principios del aprendizaje y la instrucción para la elaboración de materiales y aplicación de acciones de instrucción.

\section{IV.MODELOS CONCEPTUALES DE INSTRUCCIÓN}

Se puede decir que los modelos instrucionales son guías que brindan al docente-tutor una serie de estrategias basadas en un enfoque de aprendizaje y que ofrece los procedimientos para la realización de instrucciones, incorporando elementos fundamentales que se deben tener en cuenta en el proceso de diseño y de instrucción de un curso (Saettler, 1990). Se incluye el análisis de las metas y de los objetivos planteados, los cuales pueden ser usados en diferentes contextos al implementar el curso, generando situaciones practicas que a su vez deben ser retroalimentadas en todo momento.

Dorrego, (1991) define la instrucción como "el proceso mediante el cual se orienta el aprendizaje de un individuo, tomando en cuenta las características del que aprende y los resultados esperados del aprendizaje, así como las características y fases de este último proceso" (p.12).

Existen diferentes modelos de diseño instruccional en donde cada uno de ellos expone una idea de cómo se concibe el proceso enseñanza aprendizaje y cuáles son sus resultados al aplicarlos.

a) Modelo conductista: se fundamenta en los cambios observables de conducta y se enfoca hacia la repetición de patrones en donde el sujeto luego las realiza de manera automática. En este modelo se debe planear la estrategia de estímulos para alcanzar las respuestas deseadas y así lograr el aprendizaje, desarrollo de habilidades y competencias en el ámbito educativo.

b) Modelo cognitivista: se basa en las causas que generan los cambios de conducta, los cuales son observados para utilizarse como indicadores para entender qué pasa en la mente del que aprende. Este modelo se concentra en tres partes del proceso educativo: los objetivos del instructor, las estrategias que sigue y la inspección que hace sobre la estructura de la enseñanza.

c) Modelo constructivista: sustentado en el indicio de que cada persona construye su propia perspectiva del mundo, teniendo en cuenta sus propias experiencias y esquemas mentales desarrollados. Este modelo identifica los objetivos educativos a los cuales llegará el estudiante después de realizar una instrucción; además fomenta un proceso cognitivo en donde el estudiante puede desarrollar tareas o actividades asociadas a los objetivos educativos.

\section{FASES DEL DISEÑO INSTRUCCIONAL}

El diseño instruccional está soportado en cinco fases, a decir:

Fase 1. Análisis: se define el problema y se determinan las necesidades de la instrucción; se identifica el problema y se determinan las posibles soluciones teniendo en cuenta las características de los estudiantes. En esta fase se deben generar las metas instruccionales y realizar una lista de tareas a enseñarse.

Fase 2. Diseño: teniendo en cuenta los elementos del análisis que serán utilizados como una estrategia para producir la instrucción, se elabora un bosquejo sobre cómo alcanzar la metas instruccionales. En esta fase se debe hacer una descripción de la población, desarrollar un análisis instruccional, formular los objetivos, realizar pruebas, definir cómo se dará a conocer la instrucción y a su vez diseñar la secuencia de cómo se divulgará la información.

Fase 3. Desarrollo: se construye cada una de las unidades o lecciones y los materiales que se van a utilizar. También se elabora la instrucción, los medios que se utilizarán en la instrucción y se definirán los materiales tales como guías o talleres.

Fase 4. Implantación e implementación: en esta fase del proceso se da a conocer la instrucción, la cual se puede mostrar a través del aula virtual o de cualquier 
otro medio. Esta fase facilita la comprensión del material, da a conocer los objetivos y permite la transferencia del conocimiento del ambiente instruccional al ambiente de trabajo.

Fase 5. Evaluación: permite evaluar la instrucción de forma certera, se debe realizar en todas las etapas del proceso. Puede ser sumativa o formativa.

\section{ELEMENTOS DEL DISEÑO INSTRUCCIONAL}

El diseño instruccional tiene como objetivo transformar los cursos tradicionales usando estrategias de aprendizaje integradas y herramientas tecnológicas, para que los estudiantes puedan participar en actividades de aprendizaje colaborativas.

Los elementos del diseño instruccional se caracterizan porque al diseñarlos mantienen un ambiente de trabajo individual y grupal de manera eficiente. Los elementos son: bienvenida, introducción, intenciones educativas, objetivos generales, temario, metodología, evaluación, políticas, bibliografía, agenda de actividades, descripción de actividades y recursos de apoyo.

\section{Bienvenida}

Debe ser de carácter motivacional y personal del profesor. Puede ser un texto breve, de forma respetuosa, amable, concisa, clara y que llegue a los estudiantes.

\section{Introducción}

La introducción presenta las características del curso, sus intenciones, justificación, forma cómo se desarrollará y se evaluará el curso. En este apartado es importante presentar los datos generales del curso.

\section{Intenciones educativas}

Las intenciones educativas están relacionadas con la misión y visión del plan de estudios, establecen la relación entre el modelo de estudiante que se quiere formar y la ejecución del proceso de enseñanza y aprendizaje para alcanzar los objetivos propuestos.

\section{Objetivos}

Los objetivos se enuncian con el fin de concretar las intenciones educativas, las cuales definen la propuesta de aprendizaje.

Los objetivos deben reflejar las metas que se han planteado en el curso, se redactan en términos del alumno y se formulan de forma general y de forma específica.

\section{Temario}

Al seleccionar los contenidos del curso, se debe pensar en lo que se quiere para el trabajo de los estudiantes, teniendo en cuenta sus necesidades, sus intereses, el tiempo que durará el curso y la evaluación.

El temario da a conocer la lista de las unidades que se desarrollarán, dando la estructura a la asignatura, relacionando los objetivos, evidenciando la forma en que se desarrollará el aprendizaje y la evaluación.

\section{Metodología}

La metodología da a conocer de forma general cómo se realizarán las actividades del curso, es una guía que va indicando cómo desarrollar las actividades. La metodología debe contener elementos y estrategias de enseñanza aprendizaje, actividades a realizar de forma individual o en equipo y dar a conocer los medios para su realización.

\section{Evaluación}

Acá se expresan de manera clara los criterios que se van a tener en cuenta a la hora de evaluar el curso, la cual hace parte de un proceso permanente que se realiza antes, durante y después de la implementación del curso.

El curso suministrará evaluaciones válidas y confiables que muestren el verdadero desempeño de los estudiantes. Además los estudiantes recibirán retroalimentación específica y completa sobre cómo se ha desempeñado en el desarrollo de sus actividades de aprendizaje.

\section{Políticas}

A través de las políticas se busca dar orden al proceso de enseñanza- aprendizaje, ésta muestra la reglas que orientarán a los estudiantes para que puedan alcanzar los objetivos de aprendizaje, por lo que están encaminadas a los compromisos que adquieren tanto el estudiante como el profesor durante el desarrollo del curso.

Las políticas se clasifican en: políticas generales, compromisos del profesor, compromisos del estudiante y políticas de evaluación.

\section{Bibliografía}

Acá se presentan las fuentes de estudio que han sido empleadas como base para la construcción del curso, pero asimismo, en cada una de las unidades y/o actividades se presenta una bibliografía básica y la complementaria recomendada al estudiante para que profundice en las temáticas que se han desarrollado.

\section{Agenda de actividades}

Esta se presenta de forma organizada, mostrando los elementos: Módulo o unidad, Semana, Nombre de la actividad y Modalidad(Individual o colaborativa)

11. Descripción de actividades

El docente a través del diseño instruccional determina 
el tipo de estrategias de enseñanza y las teorías de aprendizaje que empleará para diseñar las actividades que realizarán los estudiantes, para lo cual deben tener en cuenta los contenidos a desarrollar y la evaluación del curso.

Las estrategias de enseñanza - aprendizaje adaptadas a los tipos de actividades pueden ser de autoaprendizaje, aprendizaje interactivo, aprendizaje colaborativo o estrategias integradoras.

12. Recursos de apoyo

Son los elementos que ayudarán a encontrar y mejorar las fuentes de información tales como revistas, periódicos, libros, páginas web, bibliotecas digitales, servicios de referencia virtual, tutoriales, software libre, etc.

\section{CONCLUSIONES}

El diseño instruccional orienta el quehacer docente, que lleva a reflexionar sobre los métodos utilizados en el proceso enseñanza - aprendizaje y que invita a hacer uso de las TIC como medio de comunicación eficaz en el proceso enseñanza -aprendizaje.

Se presenta como una propuesta que integra elementos disciplinares, tecnológicos, comunicacionales y pedagógicos, que se requieren para cumplir los objetivos planteados en un curso o módulo de aprendizaje.

El diseñador instruccional deberá preocuparse por proporcionar los elementos y materiales que fortalezcan el desarrollo de un curso de forma integral, buscando nuevas incursiones en el proceso de aprendizaje con aplicaciones inteligentes en la elaboración del proceso sistemático de planificación, adaptando los objetivos propuestos en el curso para llevar al estudiante a alcanzar sus metas y logros de aprendizaje de forma agradable y de calidad.

El diseñador instruccional es quien ayuda al equipo docente a hacer el enlace entre los contenidos y la tecnología para propiciar el aprendizaje, considerando las necesidades de los estudiantes, los objetivos, el modelo educativo de la institución y los recursos para diseñar e implementar un curso en línea. 三

\section{REFERENCIAS BIBLIOGRÁFICAS}

1. Aguilar, J. (2004). Diseño de instrucción, En Universidad Metropolitana, 2008. Recuperado de http://ares.unimet.edu.ve/programacion/psfase $3 /$ modll/biblio/Dlevolucion.pdf

2. Bertalanffy, L. y Weinberg. (1987). Tendencias en la teoría general de sistemas. Madrid: Alianza Editorial
3. Bertoglio, O. (1993).Introducción a la teoría general de sistemas. México: Editorial Limusa.

4. Bruner, J. (1969). Hacia una teoría de la instrucción. México: Ediciones Díaz de Santos.

5. Cervera F. (2005). Comunicación total. Madrid: Esic.

6. Chadwick, C. (1978). Tecnología educacional para el docente. Buenos Aires: Paidós.

7. Fernández D, F. y Martínez A. J. (1999): Manual básico de lenguaje y narrativa audiovisual. Barcelona: Paidós.

8. Keller, J. (1987). Development and use of the ARCS model of motivational design. Journal of Instructional Development, 10 (3), 2-10.

9. Kendall, K. E. (1991). Análisis y diseño de sistemas. México: Prentice.

10. Polo, M (2001) El Diseño Instruccional y las Tecnologías de la Información y comunicación. Venezuela: Universidad Central de Venezuela.

11. Poleo, G. (2003). Diseño Instruccional para Ambientes de Aprendizaje basados en la Web. Revista Docencia Universitaria. IV, (1) Venezuela: Universidad Central de Venezuela.

12. Serrano, J., y Pons, R. (2008). La concepción constructivista de la instrucción. Revista Mexicana de Investigación Educativa, 2008, 13 (38), 681-712.

13. Sicilia, M. (2007) Más allá de los contenidos: compartiendo el diseño de los recursos educativos abiertos. Revista Universidad y Sociedad del Conocimiento, 4 (001), 26-35.

14. Saettler, P. (1990). The evolution of american educational technology. Englewood: Libraries Unlimited, Inc.

15. Tibaná, G. y López, M. (2006). Adaptación del diseño instruccional en la construcción de ambientes virtuales de aprendizaje: caso Universidad de los Andes. Recuperado de http:// www.colombiaaprende.edu.co/html/mediateca/1607/articles-108285_archivo.pdf

16. UNESCO (1984). Glossary of Educational Technology Terms. París: Unesco.

17. Zerpa, C.(2005). El diseño de instrucción en un material educativo computarizado (MEC): La plataforma pedagógica de SIVI 1.0. Investigación y Postgrado, 20 (1), 81-113. 